\title{
The IL-10 Promoter Polymorphism at Position -592 is Correlated with Susceptibility to Occult HBV Infection
}

\author{
Behzad Nasiri Ahmadabadi, ${ }^{1}$ Gholamhossein Hassanshahi,, \\ Mohammad Kazemi Arababadi, ${ }^{1,2,4,5}$ Cristiana Leanza, ${ }^{3}$ and Derek Kennedy ${ }^{3}$
}

\begin{abstract}
Occult hepatitis B infection (OBI) is characterized as a form of hepatitis in which detectable amounts of HBV-DNA can be monitored in the peripheral blood of patients whereas the hepatitis B surface antigen is undetectable. The main aim of this study was to investigate whether there is a relationship between OBI and single nucleotide polymorphisms in the -592 region of the IL- 10 gene. In this study, the polymorphism at position -592 of the IL-10 promoter of 57 OBI cases was compared and correlated to that of 100 healthy controls by PCR-RFLP techniques. Our results showed that patient and control groups had significant differences regarding genotypes and alleles of the -592 polymorphism in the IL-10 gene. Based on our results, it can be concluded that the -592 polymorphism within the promoter of the IL-10 gene is associated with OBI.
\end{abstract}

KEY WORDS: occult hepatitis B infection; IL-10; polymorphism; HBsAg; HBV-DNA.

\section{INTRODUCTION}

Occult hepatitis $\mathrm{B}$ infection (OBI) is described as a clinical form of hepatitis $\mathrm{B}$ in which, despite the lack of detectable Hepatitis B surface antigen (HBsAg) in patient serum, those patients are positive for $\mathrm{HBV}$ DNA in periphery blood [1]. This type of hepatitis imposes a considerable threat to blood transfusion services, and its detection remains a significant challenge for those agencies [2]. Despite the application of programs for appropriate screening of all donated blood and blood components for HBsAg, some cases of posttransfusion hepatitis B are reported worldwide [3]. The

\footnotetext{
${ }^{1}$ Molecular-Medicine Research Center, Rafsanjan University of Medical Sciences, Rafsanjan, Iran

${ }^{2}$ Dept. of Microbiology, Hematology and Immunology, Faculty of Medicine, Rafsanjan University of Medical Sciences, Rafsanjan, Iran ${ }^{3}$ School of Biomolecular and Physical Science, Eskitis Institute for Cell and Molecular Therapies, Griffith University Nathan, Queensland, Australia

${ }^{4}$ Department of Microbiology and Immunology School of Medicine, Rafsanjan University of Medical Sciences, Rafsanjan, Iran

${ }^{5}$ To whom correspondence should be addressed at Department of Microbiology and Immunology School of Medicine, Rafsanjan University of Medical Sciences, Rafsanjan, Iran. E-mail: dr.kazemi@ rums.ac.ir
}

majority of post transfusion hepatitis B infections are caused by OBI [4], which we previously reported in our investigations in Isfahan [5] and Kerman [4], the two main central provinces of Iran. The mechanisms responsible for progression of $\mathrm{OBI}$ are yet to be clarified; however, some investigators have suggested that genetic and immunological parameters may play a significant role in the resistance of some individuals and sensitivity of others $[4,6,7]$. The key roles of IL-10 as an inhibitory cytokine of autoimmune and inflammatory reactions [8] raise questions concerning the impacts of this cytokine in the pathogenesis of OBI. Elevated levels of IL-10 in OBI patients were previously reported by our research team [9]. Therefore, it can be suggested that IL-10 creates an inhibitory effect on the immune system of OBI patients, and they fail to completely clear the HBV infection. Our previous findings encouraged us to explore the reasons for overexpression of IL-10 in OBI patients, with a view that if we could understand the regulatory mechanisms that are disrupted in OBI patients, this may open opportunities to explore potential therapeutics. Several studies showed that the polymorphisms within the promoter of IL-10 gene (especially -592) can influence the expression of the cytokine [10]. Therefore, the aim of 
Ahmadabadi, Hassanshahi, Arababadi, Leanza, and Kennedy

Table 1. The Table Shows the Sequence of the Primers Used in this Study as We
Product Sizes

\begin{tabular}{llc}
\hline Genes & Primers & Annealing temperature \\
\hline S gene $(\mathrm{HBV})$ & F: TCGTGGTGGACTTCTCTC & $60^{\circ} \mathrm{C}$ \\
& R: ACAGTGGGGGAAAGCCC & 500 \\
IL-10 & IL-10 -592 F: 5'-GTAATATCTCTGTGCCTC-3' & $53^{\circ} \mathrm{C}$ \\
& IL-10 -592 R: 5'-CATTCCAGAATACAATGG-3' & \\
\hline
\end{tabular}

this study was to investigate the relation between OBI and the -592 polymorphism of IL-10 gene promoter.

\section{MATERIAL AND METHODS}

\section{Patients}

Peripheral blood samples were collected from 57 OBI patients and 100 healthy controls. HBsAg-negative and HBV-DNA/anti-HBc-positive samples were considered as OBI, while, HBsAg/HBV-DNA-negative, and anti-HBc-positive samples were selected as healthy controls. Selection of OBI patients and healthy controls was described previously [4]. The study protocol was approved by the ethical committee of the Rafsanjan University of Medical Sciences.

Prior to sample collection, all participants of this study filled out and signed the informed consent form which was designed and based on the aims and objectives of the current study.

\section{Genomic DNA Extraction}

Peripheral blood was collected on EDTA, and genomic DNA was extracted using a commercial kit (Bioneer, Korea) following the manufacturer's recommended procedures. Extracted DNA was aliquoted (for each patient sample) and stored at $-20^{\circ} \mathrm{C}$ for further use.

Table 2. Frequency of Polymorphisms within the -592 Region of the IL-10 Gene in OBI Patients and Controls

\begin{tabular}{llcl}
\hline $\begin{array}{l}\text { Condition } \\
\text { Genotype }\end{array}$ & Patients & Control & p value \\
\hline $\mathrm{C} / \mathrm{C} n(\%)$ & $31(54.4 \%)$ & $22(22 \%)$ & $p=0.001$ \\
$\mathrm{~A} / \mathrm{C} n(\%)$ & $24(42.1 \%)$ & $55(55 \%)$ & \\
$\mathrm{A} / \mathrm{A} n(\%)$ & $2(3.5 \%)$ & $23(23 \%)$ & \\
$\mathrm{Alleles}$ & & & \\
$\mathrm{C} n(\%)$ & $86(75.44 \%)$ & $99(49.5 \%)$ & $p=0.001$ \\
$\mathrm{~A} n(\%)$ & $28(24.56 \%)$ & $101(50.5 \%)$ & \\
\hline
\end{tabular}

\section{Detection of Polymorphism}

78

The -592 IL-10 gene polymorphism (within the gene promoter) was analyzed by the PCR-RFLP method as described in our previous study [11].

\section{Statistical Analysis}

Hardy-Weinberg equilibrium was assessed using the genotype data. Allele and genotype frequencies were calculated in patients and healthy controls by direct gene counting. Statistical analysis of the differences between groups was determined by the $\chi^{2}$ test using EPI 2000 and SPSS software version 13. A $P$ value of less than 0.05 was considered significant.

\section{RESULTS}

Evaluation of polymorphisms within the -592 region of the IL-10 gene by $R$ sa-1 restriction digestion showed that the prevalence of the $\mathrm{C} / \mathrm{C}$ genotype was $31(54.4 \%)$ in patients and $22(22 \%)$ in controls, the frequency of the $\mathrm{A} / \mathrm{C}$ genotype was 24 $(42.1 \%)$ and $55(55 \%)$ in patients and controls, respectively, and the values for the A/A genotype in the patient group was $2(3.5 \%)$ and in controls was $23(23 \%)$ (Table 1). Statistical analysis showed a significant difference between groups regarding these genotypes $(p=0.04)$. The frequency of the $\mathrm{C}$ allele was $86(75.44 \%)$ and $99(49.5 \%)$ in patients and controls, respectively. Twenty-eight $(24.56 \%)$ of the A alleles were seen in patients, but the frequency of this allele was 101 (50.5\%) in controls. Statistical analysis showed that the difference in these genotypes were also significant $(p=0.001)$ (Table 1$)$.

\section{DISCUSSION}

Increased serum levels of IL-10 subsequent to the viral infections is now well documented [9], and it has
108

79 80 81 82 83 84 85 86 87 88 89

91 92 93 94 95 96 97 98 99 100 101 102 103 104 105 106 107 


\section{IL-10 Polymorphism in Occult HBV Infection}

111

112

Q1 113

114

115

116

117

118

119

120

121

122

123

124

125

126

127

128

129

130

131

132

133

134

135

136

137

138

139

140

141

142

143

144

145

146

147

148

149

150

151

152

153

154

155

156

157

158

159

160 also been reported that the expression level of IL-10 is related to the type of clinical presentation and stage of hepatitis B virus infection (Table 2). In addition, IL-10 levels have also been associate with relevant liver disease [12]. In agreement with the evidence suggesting a potential correlation between IL-10 and disease status, our results showed that the frequency of evaluated alleles and genotypes were different between OBI patients and healthy controls. Therefore, based on our results, it can be concluded that these polymorphisms are associated with OBI. In a previous study, we reported the overexpressed circulating levels of IL-10 in OBI patients [9]; hence, when considering these data together, it might be concluded that the evaluated polymorphisms probably had an impact on IL-10 production. To the best of our knowledge, this is the first study to evaluate the IL-10 -592 polymorphisms in OBI patients. However, several studies have shown that IL-10 polymorphisms correlate with hepatitis B [13-15]. For instance, LU Yong-Liang reported that the polymorphisms at the -592 position of the IL-10 gene were associated with HBV infection in an Asian ethnic group [13]. Interestingly, they did not find any relationship between HBV infection and any other polymorphisms found within the IL-10 promoter [13]. Some studies also demonstrated that the polymorphisms at the -592 position of IL-10 are associated with HBV infection [15, 16]. Interestingly, a meta-analysis showed that the frequency of A allele at the -592 position of IL-10 is more likely to be related to spontaneous HBV clearance [17]. In this study, we revealed that the frequency of $\mathrm{A}$ allele was decreased in the OBI patients; thus, it can be concluded that the evaluated IL-10 polymorphisms in OBI patients may lead to a weakened immune system which fails to clear HBV. Interestingly, our previous study showed that the serum levels of IL-12 were decreased in OBI patients [4]. Additionally, we showed in that study that the polymorphisms within IL-12 were not associated with IL-12 serum levels [4]. Therefore, according to our current and previous findings, it may be concluded that low levels of inflammatory cytokines such as IL-12 are related to the inhibitory effects of IL-10.

The strength of our study is the high number of the evaluated blood donors. However, there is a lack of functional correlation between the presence of the polymorphism and its role in regulating IL-10 expression. In the future, this could be resolved using a luciferase reporter assays in which reporter expression is studied under the control of the wild-type and diseased promoters. In addition, future studies should focus on the expression levels of IL-10 mRNA in the immune cells of OBI patients versus healthy controls by real-time PCR. It would also be interesting to measure circulating serum levels of IL-10 in OBI patients carrying the -592 polymorphism versus healthy controls.

Finally, due to the complexity of OBI, other aspects of the disease need to be examined. Therefore, our future studies will be focused on exploring polymorphisms and the expression levels of related cytokines and their receptors in OBI patients.

\section{ACKNOWLEDGMENT}

The authors of this article would like to take this chance to acknowledge all the OBI patients and healthy controls who contributed to this research; your input is appreciated. This work was supported by a grant from the Rafsanjan University of Medical Sciences.

172

173

174

175

176

\section{REFERENCES}

1. Hollinger, F.B., and G. Sood. 2009. Occult hepatitis B virus infection: a covert operation. Journal of Viral Hepatitis 17: 1-15.

2. Schmeltzer, P., and K.E. Sherman. 2010. Occult hepatitis B: clinical implications and treatment decisions. Digestive Diseases and Sciences 55: 3328-3335.

3. Candotti, D., and J.P. Allain. 2009. Transfusion-transmitted hepatitis B virus infection. Journal of Hepatology 51: 798-809.

4. Arababadi, M.K., A.A. Pourfathollah, A. Jafarzadeh, G. Hassanshahi, S. Daneshmandi, A. Shamsizadeh, and D. Kennedy. 2011. Nonassociation of IL- $12+1188$ and IFN-gamma +874 polymorphisms with cytokines serum level in occult HBV infected patients. Saudi Journal of Gastroenterology 17: 30-35.

5. Pourazar, A., M. Salehi, A. Jafarzadeh, M.K. Arababadi, F. Oreizi, and K. Shariatinezhad. 2005. Detection of HBV DNA in HBsAg Negative Normal Blood Donors. Iranian Journal of Immunology 2: 172-176.

6. Zerbini, A., M. Pilli, C. Boni, P. Fisicaro, A. Penna, P. Di Vincenzo, T. Giuberti, A. Orlandini, G. Raffa, T. Pollicino, G. Raimondo, C. Ferrari, and G. Missale. 2008. The characteristics of the cellmediated immune response identify different profiles of occult hepatitis B virus infection. Gastroenterology 134: 1470-1481.

7. Demir, M., E. Serin, S. Gokturk, N.A. Ozturk, S. Kulaksizoglu, and U. Ylmaz. 2008. The prevalence of occult hepatitis B virus infection in type 2 diabetes mellitus patients. European Journal of Gastroenterology and Hepatology 20: 668-673.

8. Sanjabi, S., L.A. Zenewicz, M. Kamanaka, and R.A. Flavell. 2009. Anti-inflammatory and pro-inflammatory roles of TGF-beta, IL-10, and IL-22 in immunity and autoimmunity. Current Opinion in Pharmacology 9: 447-453.

9. Arababadi, M.K., A.A. Pourfathollah, A.A. Jafarzadeh, and G. Hassanshahi. 2010. Serum levels of Interleukin (IL)-10 and IL-17A in occult HBV infected south-east Iranian patients. Hepatitis Monthly 10: 31-35.

10. Karjalainen, J., J. Hulkkonen, M.M. Nieminen, H. Huhtala, A. Aromaa, T. Klaukka, and M. Hurme. 2003. Interleukin-10 gene promoter region polymorphism is associated with eosinophil count
178

179

180

181

182

183

184

185

186

187

188

189

190

191

192

193

194

195

196

197

198

199

200

201

202

203

204

205

206

207

208

209

210

211 
and circulating immunoglobulin $\mathrm{E}$ in adult asthma. Clinical and Experimental Allergy 33: 78-83.

11. Arababadi, M.K., M.R. Mirzaei, S.M.A. Sajadi, G. Hassanshahi, B. N. Ahmadabadi, V.A. Salehabadi, R. Derakhshan, and D. Kennedy. 2011. Interleukin (IL)-10 gene polymorphisms is associated with type 2 diabetes with and without nephropathy: a study of patients from the South-East region of Iran. Inflammation, in press.

12. le Song, H., V.Q. Binh, D.N. Duy, J.F. Kun, T.C. Bock, P.G. Kremsner, and A.J. Luty. 2003. Serum cytokine profiles associated with clinical presentation in Vietnamese infected with hepatitis B virus. Journal of Clinical Virology 28: 93-103.

13. Lu, Y.L., X. Wu, H.L. Huang, and L.C. Dai. 2010. Allele polymorphisms of interleukin-10 and hepatitis B, C virus infection. Chinese Medical Journal (Engl) 123: 1338-1344.

14. Gao, Q.J., D.W. Liu, S.Y. Zhang, M. Jia, L.M. Wang, L.H. Wu, S.Y. Wang, and L.X. Tong. 2009. Polymorphisms of some cytokines and chronic hepatitis B and C virus infection. World Journal of Gastroenterology 15: 5610-5619.
15. Wang, C.J., K.R. Shan, Y. He, T. Zhang, Y. Li, X.L. Qi, Y. Zhao, Y. Xiao, C.X. Wu, Z.Z. Guan, and X.L. Ren. 2008. Study on the association of IL-10 -592 polymorphism with susceptibility to hepatitis B viral infection in Han, Yi and Yao ethnic groups in Guizhou province. Zhonghua Liu Xing Bing Xue Za Zhi 29: $444-448$.

16. Miyazoe, S., K. Hamasaki, K. Nakata, Y Kajiya, K. Kitajima, K. Nakao, M. Daikoku, H. Yatsuhashi, M. Koga, M. Yano, and K. 238 Eguchi. 2002. Influence of interleukin-10 gene promoter poly- 239 morphisms on disease progression in patients chronically infected 240 with hepatitis B virus. American Journal of Gastroenterology 97: 241 2086-2092.

17. Zhang, T.C., F.M. Pan, L.Z. Zhang, Y.F. Gao, Z.H. Zhang, J. Gao, 243 R. Ge, Y. Mei, B.B. Shen, Z.H. Duan, and X. Li. 2010. A meta- 244 analysis of the relation of polymorphism at sites -1082 and $-592 \quad 245$ of the IL-10 gene promoter with susceptibility and clearance to 246 persistent hepatitis B virus infection in the Chinese population. 247 Infection 39: 21-27. 